Théologiques

Théologiques

\title{
Territoire et sacré : penser un lien millénaire à l'aune du XXI siècle
}

\section{Michel Beaudin et Marie-Joëlle Zahar}

Volume 16, numéro 1, 2008

Le territoire et le sacré

URI : https://id.erudit.org/iderudit/019182ar

DOI : https://doi.org/10.7202/019182ar

Aller au sommaire du numéro

Éditeur(s)

Faculté de théologie et de sciences des religions, Université de Montréal

ISSN

1188-7109 (imprimé)

1492-1413 (numérique)

Découvrir la revue

\section{Citer ce document}

Beaudin, M. \& Zahar, M.-J. (2008). Territoire et sacré : penser un lien millénaire à l'aune du XXI ${ }^{\mathrm{e}}$ siècle. Théologiques, 16(1), 5-23.

https://doi.org/10.7202/019182ar d'utilisation que vous pouvez consulter en ligne.

https://apropos.erudit.org/fr/usagers/politique-dutilisation/ 


\title{
Territoire et sacré : penser un lien millénaire à l'aune du $\mathrm{XXI}^{\mathrm{e}}$ siècle
}

\author{
Michel BEAUDIN* \\ Faculté de théologie et de sciences des religions \\ Université de Montréal \\ Marie-Joëlle ZAHAR* \\ Département de science politique \\ Université de Montréal
}

Cette livraison de Théologiques est consacrée à la question des rapports entre le territoire et le sacré, un problème qui paraît aussi ancien que les sociétés, mais qui resurgit avec une actualité aiguë, sinon inattendue, en ce début de $\mathrm{XXI}^{\mathrm{e}}$ siècle, dans un contexte qui le remodule et oblige à le penser à nouveaux frais. Nous évoquerons d'abord comment le "territoire» et le "sacré » se retrouvent dans le tourbillon de cette actualité, tout en précisant provisoirement ce qu'on peut entendre par ces termes. Nous soumettrons ensuite quelques considérations sur l'approche de la question et des différentes configurations du rapport territoire-sacré, lesquelles guideront, enfin, la présentation des contributions de cinq auteurs d'horizons divers à cette réflexion.

* Michel Beaudin est professeur honoraire à la Faculté de théologie et de sciences des religions de l'Université de Montréal. Ses recherches actuelles portent sur la théologie contextuelle québécoise et sur des perspectives théologiques et anthropologiques pour une alternative au néolibéralisme comme «salut» sélectif ou sacrificiel. Il a publié en 2006: «Le vivre ensemble, entre sacrificialité et solidarité», dans G. Vincent, dir., Hospitalité et solidarité. Éthique et politique de la reconnaissance, Strasbourg, Presses universitaires de Strasbourg.

* Marie-Joëlle Zahar est professeur agrégé au Département de science politique de l'Université de Montréal. Ses recherches actuelles portent sur les interventions internationales et la construction de la paix au Liban, ainsi que sur le pouvoir et le rôle des citoyens au Moyen-Orient. Elle a publié en 2008, dans la revue Sudosteuropa: «Power Sharing, Credible Commitment, and State (Re-)Building: Comparative Lessons from Bosnia and Lebanon». 


\section{1. «Territoire" et "sacré»: clarifications sémantiques autour d'un vieux problème toujours aussi explosif}

Les enjeux "territoriaux» ont toujours partie liée avec quelque forme de "sacré ». Toutefois, et pour fin d'analyse, nous aborderons d'abord séparément ces deux notions, avant de considérer leurs rapports.

Mais ne faut-il pas rappeler auparavant le rôle central joué par la convoitise et les luttes pour la répartition des territoires dans l'histoire de l'humanité ? Que l'on pense ainsi, pour l'Antiquité, à la constitution de l'Empire romain puis à sa dissolution sous la pression des «Barbares ». Et pour le Moyen-Âge, aux guerres féodales et aux Croisades suivies des guerres de religions en Europe. Puis aux conquêtes du Nouveau Monde et aux autres processus de colonisation qui s'ensuivirent, dont le découpage de l'Afrique en territoires correspondant si peu à ceux des ethnies qui y étaient établies et aux conséquences encore très vives. Et finalement, à ce $\mathrm{XX}^{\mathrm{e}}$ siècle des deux grandes Guerres mondiales, de la Guerre froide et de ses affrontements par pays du Tiers-Monde interposés, des luttes de décolonisation, de l'après-Guerre froide faisant resurgir des guerres civiles. Toujours et partout, des conflits de territoire égrenant à l'infini leur chapelet de souffrances pour les populations touchées.

Si la fin de la Guerre froide a initialement suscité l'attente selon laquelle la fin de l'histoire (Fukuyama) et la victoire de la démocratie ouvriraient la voie à une ère de paix kantienne - soit l'obsolescence de la guerre (Mueller 1989) —, les développements des quinze dernières années ont mis à mal les théories et les espoirs qui voulaient que le troisième millénaire, du haut de son observatoire de l'histoire, de ses conquêtes démocratiques, de sa modernité, de ses institutions internationales, de la mondialisation, ou encore de sa conscience des menaces environnementales, permette, enfin, à une humanité fatiguée de souffler un peu.

Si ce rappel du passé a pu paraître superflu, on ne pourra en dire autant du caractère impérieux d'une réflexion sur les dynamiques territoriales au regard de l'orage qui monte encore à l'horizon.

À l'heure de la "glocalisation ", de l'imbrication du local et du global, de la mondialisation sous le signe du néolibéralisme et des résistances qui lui sont opposées, la question du territoire demeure omniprésente dans les discours publics, que ceux-ci soient à teneur normative ou politique, sociale ou économique. De Jérusalem au Kosovo, du Darfour au Tibet, des «réserves indiennes » trop exiguës d'Amérique du Nord aux bidonvilles du Tiers-Monde, des migrations volontaires aux déplacements forcés de 
population, des luttes contemporaines autour de la souveraineté étatique à la mobilisation écologique pour la sauvegarde de la planète, ou encore de la "conquête des marchés » au nom de la libéralisation économique à la nouvelle course pour le partage des ressources pétrolières et gazières de l'Arctique aux frontières mal définies, et sans parler des incidences territoriales obvies de la "guerre au terrorisme ", conflits et revendications ne cessent de référer au rapport à l'espace territorial, sinon d'en faire leur enjeu principal. La terre s'est rétrécie sans que la sagesse collective se soit élevée à la hauteur des conséquences prévisibles de ce changement pour le vivre ensemble des peuples, inséparable de la paix territoriale.

\section{«Territoire»}

Que faut-il entendre par "territoire» ? Dans le cadre d'une problématique sommaire de notre thème présentée initialement aux auteurs pour les aider à situer leurs contributions respectives, nous leur avions soumis les points de repères suivants.

Dans la tradition plus ancienne, «le territoire renvoie à la localisation d'un effet de pouvoir ». Il réfère à un découpage politique et juridictionnel de la surface terrestre en termes de souveraineté. Ainsi, la souveraineté territoriale moderne (gouvernement et peuple associés historiquement à une terre spécifique) s'est développée en partie en réaction à la souveraineté personnelle, féodale et royale. "Cette relation entre territoire et pouvoir opère dès lors tant à l'intérieur [...] qu'à l'extérieur (relations entre divers territoires)». Le «territoire (le plus souvent désigné comme national) se rapporte au domaine de la souveraineté et informe comme tel la réflexion en géographie politique et en géopolitique» (Dory 1989, 2574).

La seconde configuration, liée à l'éthologie d'abord, puis à l'écologie humaine et à l'anthropologie écologique, définit le territoire comme "une aire occupée plus ou moins exclusivement par un individu ou un groupe au moyen du refoulement opéré par un comportement agressif ou par l'émission d'un signal quelconque " (Dory 1989, 2574). Cette définition n'est donc pas étrangère à la configuration proprement politique, qui en constitue comme un cas d'espèce.

Dory fait aussi état de nouveaux développements de la réflexion. On cherche ainsi à "cerner la matrice anthropologique de l'action géographique d'appropriation/transformation de la nature ». Le territoire se présente, enfin, à la fois comme condition (ressource) et comme résultante (organisation économique, politique, symbolique, etc.) du procès social. De même, 
la géographie culturelle s'intéresse à l'ensemble des représentations dont le territoire fait l'objet, ce qui touche directement notre thématique. Enfin, on fait jouer à la notion de territoire un rôle critique par rapport à la notion d'espace, entre autre par le biais de l'interface des dialectiques de la nature et de la société $(1989,2575)$.

Retenons au total, ici, que le "territoire ", tant au sens propre que métaphorique, désigne à la fois les dimensions géographique (ou spatiale) et sociale d'un processus référant d'abord à une démarcation ou délimitation de type politique, mais non exclusivement. Car, comme nous le verrons plus loin, la globalisation économique se redéfinit maintenant une territorialité propre, coïncidant non seulement avec les dimensions mêmes de la planète, mais aussi avec l'ensemble de la réalité et des activités humaines, désormais potentiellement visées par la marchandisation. À l'opposé, l'altermondialisme revalorisera, sans s'y enfermer, le territoire local, et selon une autre logique de développement, plus centrée sur le lien social.

\section{Le «sacré»}

Mais voici que c'est habituellement à un titre ou à un autre que l'humain a historiquement revendiqué le territoire sur lequel il vivait ou qu'il convoitait. Par exemple, en tant que legs divin, comme dans les notions de «terre promise »; en tant que droit national forgé dans la longue durée et le sang, comme dans le discours sur l'État-nation; ou encore au titre d'un droit personnel (ou de propriété) acquis par le biais de l'utilisation productive de la terre (Locke 1990), etc. Les titres sont souvent multiples, mais avec une dominante. Celle-ci sera parfois religieuse. Ainsi, être juif, chrétien ou musulman c'est, au-delà de tout corpus théologique, une relation particulière à des lieux: la terre d'Israël, Jérusalem, Nazareth ou Bethléem, ou encore La Mecque et Médine. Elle peut également être nationale. Là encore, plusieurs communautés peuvent se réclamer d'un même territoire à des titres divers. C'est ainsi que le Kosovo, où la majorité d'origine albanaise a développé un nationalisme propre, est aussi revendiqué par la nation serbe, car ce territoire se trouve au cœur de son mythe fondateur, la bataille de Kosovo Polje en 1389. Lieux sacrés, lieux partagés, lieux contestés.

Dans tous les cas semble intervenir un troisième terme entre le revendicateur et tout contestateur possible : ce «au nom de » quoi ou de qui pourrait se légitimer cette revendication d'un territoire. Quelle que soit l'instance en cause, la démarche de revendication implique un processus pouvant mener à la sacralisation tant de la référence que du territoire lui-même. 
Cette observation est à la source de la présente thématique. Mais avant d'en explorer davantage le bien-fondé ou non, avançons quelques précisions cette fois sur la notion de "sacré ». Un très bref aperçu proposé à la réflexion des auteurs se basait sur un article de l'Encyclopédie philosophique universelle (Texier 1989, 2291-2293). L'essentiel en a été repris par Houtart. Nous y ferons référence puis nous expliciterons en faisant appel à d'autres voix.

S'il est une idée sur laquelle les spécialistes semblent s'accorder à propos de la signification du "sacré ", c'est celle de séparation, exprimée par le radical sak qu'on trouve en plusieurs langues (hittite, germanique, latine, grecque) et dont vient aussi le mot sanctus (Texier 1989, 2291). La distinction la plus classique serait la bipartition entre le sacré et le profane ${ }^{1}$ qu'on trouve, par exemple, dans toute l'histoire des Grecs (Despland 1979, 9). Entre les deux, il y a d'abord une différence, une juxtaposition sans mélange possible. Ce qui ne veut pas dire qu'il n'y ait pas de communication ou de passage entre les deux "mondes ", sinon le sacré cesserait d'être relatif au profane et de lui être essentiel. Le culte sert aussi à ce passage, dans les deux sens, d'ailleurs.

Par ailleurs, si des divinités sont spontanément rangées du côté du sacré, des personnes (rois, prêtres...), des lieux (sanctuaires, terre...), des objets (autels, victimes, vêtements...) ou des moments (jours fériés, semaine sainte, mois du Ramadan...) peuvent aussi être « consacrés ». Durkheim dira : " un riche, un arbre, une source, un caillou, une pièce de bois, une maison, en un mot, une chose quelconque peut être sacrée » $(1968,51)$. Il y a là l'idée d'un retrait de la circulation, d'une mise à part en direction d'une transcendance ou du divin, dont découle la reconnaissance d'une valeur intangible et d'une inviolabilité en raison du caractère acquis (Texier 1989, 2291).

Pour Durkheim, le sacré est marqué par l' "ambiguïté ». Il parle de forces religieuses partagées entre puissances bienfaisantes et puissances maléfiques, antagonisme organisateur de la polarité entre le pur et l'impur ou entre le faste et le néfaste. Il s'est étonné que des choses puissent passer d'un pôle à l'autre selon les circonstances ${ }^{2}$, mais "sans changer de nature ". Constatant que dans un cas comme dans l'autre, "une communion des consciences » et un « réconfort mutuel » sont produits, il en vient à conclure

1. Séparation symbolisée, en latin, par les deux espaces du fanum (temple) et du profanum (les abords du temple).

2. Par exemple, qu'un mort cesse d'être craint pour devenir un génie protecteur une fois les rites et la période de deuil terminés. 
que ces puissances sacrées, tutélaires ou malfaisantes, correspondent à des "états collectifs objectivés ", qu'elles "résultent de la vie collective et l'expriment ", qu'elles représentent la société saisie dans des "attitudes » différentes, la vie sociale, dans son unité et sa diversité (Durkheim 1968, 584-591). Selon Malise Ruthven, Durkheim « equated the sacred with the spirit of the community, a projection of the communal spirit onto a supernatural, transcendantal Being " $(2004,153)$. Lui-même confirme bien ce jugement, mais en insistant finalement sur le sens ou la visée de ce processus. Pour lui, les pratiques et les croyances ne sont pas à ranger dans «des genres séparés ». Il les réunit dans la «vie religieuse» qui a "pour objet d'élever l'homme au-dessus de lui-même et de lui faire vivre une vie supérieure à celle qu'il mènerait s'il obéissait uniquement à ses spontanéités individuelles: les croyances expriment cette vie en termes de représentation; les rites l'organisent en en réglant le fonctionnement» $(1968,592)$.

D'une part, en ce qui concerne l'ambivalence du sacré, nous sommes ici tout près de René Girard qui, après le transfert d'agressivité collective sur le bouc émissaire, conçoit la sacralisation de la victime comme la résultante d'un transfert de réconciliation attribuée à celle-ci (Girard 1978, 45). D'autre part, chez lui comme chez Durkheim, on trouve cette fonctionnalité du sacré, chez le premier comme matrice de la religion et de la culture, et chez le second comme médiation indispensable à la cohérence de la vie en société ou du moins à la dynamique de son "progrès ». Les deux, également, présentent les rites, — dont le sacrifice - , comme le pendant du sacré au niveau de l'organisation et de la régulation de la société. Mais nous sommes déjà là dans le rapport entre le territoire et le sacré auquel nous allons maintenant nous attarder, négligeant ici l'expérience subjective du sacré, analysée par Otto (1949, en fr.), bien que nous soyons conscients que le sujet reste au centre de la construction de tout sacré.

\section{Vers une problématisation du rapport entre le territoire et le sacré}

Comment penser ce rapport? Nous avancerons ici quelques pistes bien fragmentaires, mais qui pourraient stimuler la lecture et l'interprétation des textes de ce numéro. Ces pistes couvrent tant la sacralisation du territoire que la territorialisation du sacré, processus constitutifs du rapport entre le sacré et le territoire, qui s'enchevêtrent, se renforcent mutuellement et peuvent, dans certains cas précis, paraître quasiment inextricables. 


\section{Sacralisation du territoire et rapport à l'autre}

En nous limitant d'abord au processus de constitution d'un territoire singulier, nous proposons au lecteur et à la lectrice d'y voir une figure particulière de la structure anthropologique du rapport à l'autre proposée par le philosophe Dany-Robert Dufour. Celui-ci émet l'hypothèse selon laquelle l'identité passe par un rapport de pleine reconnaissance de l'autre, rapport qui ne serait rendu possible que par une Référence commune, ou Tiers, qui à la fois re-présente, fonde et se porte garant de ce lien social entre l'un et l'autre, ou du nous ainsi formé (1990, 15-69). Rapport structuré selon une logique ternaire, donc, mais qui ne cesse d'être soumis aux possibles dérives de types unaire ou binaire, ou même de se dissoudre dans l'immédiateté (Dufour 2003, 15-17), comme Houtart l'a bien décrit puis illustré dans sa contribution. Socialement et politiquement, le territoire, c'est le rapport à l'autre constitué en vivre ensemble sous une certaine identité, en communauté. Structurellement, le sacré, s'il y a lieu, y joue le rôle de Tiers ou de Référence.

On pourra rapprocher de Dufour la perspective de Pierre Legendre à propos d'une «coupure [...] qui permet à l'homme d'habiter le monde, parce que le monde lui est re-présenté, présenté à nouveau » $(1998,66)$. Tel un invariant, précise-t-il, "la scène de la vérité ne disparaît jamais " $(1998,70)$. Que le sacré apparaisse comme divin ou donné des dieux, dans les sociétés traditionnelles ou dans certaines religions actuelles, tel le christianisme, ou encore comme consciemment construit dans l'artificialisme moderne, la structure reste la même. Citons encore Legendre $(1998,69)$ :

Dans les sociétés tenues par le ciment religieux [...] Dieu fait tenir l'ensemble. Mais dans l'hypothèse des sociétés laïcisées, succédant à cet état historique, qu'en est-il ? Comment opère l'équivalent du divin, voire son substitut, là où le Texte se donne comme ayant congédié la Tradition? Nous présentons ce à quoi en définitive nous avons affaire: circonscrire et définir le principe du garant.

Prenant l'exemple d'un texte sacré, quel qu'il soit, il dit: « Nous l'appellerons sacré, selon le sens originel du mot, ce qui est consacré aux dieux, ou encore, en termes conformes au fonctionnement sécularisé des sociétés ultramodernes, consacré à la Référence fondatrice » $(1998,70)$.

Avec la séparation de l'Église et du politique, propre aux Lumières, on voit naitre une nouvelle logique ou forme de sacralisation du pouvoir. Celuici n'est plus divin ou de droit divin, mais bien humain. Il est toutefois 
«transcendant» en ce sens qu'il représente non pas un ou plusieurs individus, mais ce corps politique qu'est la «nation». Nous retiendrons ici le point de vue qui définit la nation comme une communauté imaginée construite autour d'un lien généalogique plus ou moins fictif — impliquant un vernaculaire et un passé commun - et rattachant la communauté en question à un lieu particulier (Anderson 1993). Un corps, donc, mais qui, dans son unité, se transforme et devient bien plus que la simple somme de tous ses membres. Ainsi transfigurée, la nation prend corps : c'est une communauté au sens de Benedict Anderson, "un être collectif "sui generis" doté d'une volonté propre: la "volonté générale" » (Patez 1998) et qui ainsi donne une personnalité à l'État, comme l'illustre la figure du Léviathan qui orne le célèbre ouvrage de Hobbes du même nom.

F. Ost exprime le même rapport dans les termes plutôt de l'« hétéroréférence » dont a besoin l'autonomie pour s'épanouir, de la dialectique de la loi et du contrat, de "l'imposé et du négocié ». Il s'agit de «penser ensemble initiative des acteurs et principes régulatoires" dans une "négociation" — non réductible "au seul "moyen terme" des rapports de forces ou d'intérêts en conflit» — ou engendrement réciproque apte à produire «l'instance tierce - le tiers - seule en mesure d'opérer la médiation de la relation» (Ost 1999, 7). L'hétéro-référence, ou transcendance, n'est donc pas entendue ici «exclusivement comme la position d'une extériorité » mais aussi comme «faculté d'auto-dépassement du social » (Ost 1999, 14-19) ou d' « auto-transcendance » pour emprunter à J.-P. Dupuy $(1988,172$ s) auquel réfère Ost. Celle-ci est "produite par le jeu du social lui-même, mais à la manière d'un point fixe endogène et pourtant structurant pour cette société »(Ost 1999, 20). Ni "autodétermination à la fois transparente à elle-même et auto-suffisante ", ni "programmation externe qui se traduirait par la dépendance la plus complète et l'opacité intégrale du social à luimême» (1999, 19). Pour sa part, Cosmao exprime ainsi cette nécessaire coupure (non absolue, évidemment) entre la communauté concrète et sa référence, même dans le cas du pouvoir explicitement socialement construit: «il ne s'impose aux hommes qu'en échappant à leur maîtrise » $(1981,47)$.

Sur le versant économique, plus précisément dans l'ordre marchand moderne - ou capitalisme - un certain nombre de règles et d'institutions, appelées "marché », tiennent lieu de référence sacrée. En son sein, les prix agissent comme instance régulatrice, et la monnaie, ce bien mis à part, sert concrètement de tiers commun, d'équivalent général, ainsi que de mesure 
et de garantie des échanges (Aglietta et Orléan 1982, 40-49). Mais voici qu'en ces temps de néo-libéralisme, particulièrement, c'est la monnaie ellemême qui se transforme en marchandise et fait l'objet d'échanges spéculatifs (marché des devises), ce qui a pour effet de dérégler l'économie en l'abandonnant à une sorte d'auto-référencialité. Le territoire de l'économie se met alors à échapper à son indispensable maîtrise par la société, ce qui inaugure la destruction «économique » de celle-ci. En effet, non seulement la teneur du marché comme tiers est-elle binaire et donc facteur de « déliaison " sociale par une compétition à outrance, mais, cessant d'agir comme véritable référence, le marché remet les sociétaires à l'immédiateté de leurs rapports de force. Une «société de marché » n'a pas d'avenir humain.

Pour clore sur ce premier aspect fondamental du rapport entre le territoire et le sacré, ajoutons ceci : ce qui vaut pour la cohésion interne d'un territoire donné, soit l'existence et la reconnaissance commune d'une référence de sens représentative, vaut aussi pour le lien à d'autres territoires, que ce soit entre des peuples au sein d'un État fédéral ou confédéral, à un plan interétatique comme dans la Communauté européenne, ou au sein du système des Nations unies. Celui-ci, par exemple, est basé sur les Étatsnations. Est reconnu comme État, selon le droit international, tout territoire délimité où vit une population encadrée par un gouvernement effectif qui exerce sa souveraineté et dont le reste des États reconnaissent la légitimité. La pierre angulaire du système international, c'est la souveraineté, "sacralisée » par les règles établies lors de la création du Concert de l'Europe et institutionnalisées dans le cadre de l'Organisation des Nations unies, au lendemain de la Deuxième Guerre mondiale.

Dans tous les cas, ce lien interterritorial, en supposant qu'il respecte aussi chaque identité territoriale, ne pourra se forger que par la recherche et la construction de références communes prenant alors un statut s'apparentant au moins au sacré. Autrement, le rapport risquera toujours de dériver vers l'antagonisme comme un romancier l'a bien mis en scène récemment et de façon extrême entre "Globalia » et les "non-zones " (Rufin, 2004). Il est intéressant, en ce sens, de noter la similitude frappante entre la manière dont les sociétés traditionnelles perçoivent leur "Monde ", espace sacré, contrairement au «Chaos » qui s'étend au-delà de ses frontières, et la manière dont les États du système international perçoivent toute attaque contre leur souveraineté (Eliade 1969, 47). Dans les deux cas, le vocabulaire tend à associer le "Monde" avec les forces du bien, et le "Chaos» avec celles du mal, plusieurs des nationalismes contemporains 
partageant ainsi avec les processus traditionnels de délimitation sociopolitique une logique binaire basée sur une notion négative de "l'autre».

\section{Territorialisation du sacré}

L'autre volet de cette problématique abordera la question de la teneur de la référence fondatrice et celle de l'articulation entre le rapport à celle-ci et les rapports au sein du territoire. Nous serons très brefs, ici, car Houtart traite de l'essentiel de ces aspects en introduction de son article.

On aura compris que le «sacré » recouvre des références ou des garants de degrés variables en termes d'intensité de cette fonction jusqu'à une connotation divine ou religieuse, avec des effets correspondants de saisissement chez ceux qui s'en réclament. La "sacralisation» de la référence exprime généralement son durcissement ou la levée de toute limite. Nous considérons qu'il se produit alors deux choses. D'une part le Tiers se ferme sur lui-même au lieu d'ouvrir à une référence plus haute, ce qui l'apparente alors à une idole. D'autre part, l'autre indice d'une sacralisation idolâtrique est que le Tiers ne renvoie plus à la reconnaissance et à la promotion de l'autre. Le Tiers cesse alors d'être "relatif ", au sens d'avoir une portée limitée et non absolue, et au sens d'être au service du vivre ensemble à vivifier. Au moins peut-on se demander si le besoin d'un Tiers pour unir et créer une identité nécessite de le sacraliser, et dire, avec Durkheim, que la sacralisation fait verser dans l'ambiguité.

Après avoir observé comme une constante historique que «les sociétés ne se structurent qu'en sacralisant leurs normes », Cosmao exprime bien, dans un corollaire, ce dévoiement de la référence: "plus les sociétés se structurent dans l'inégalité, plus elles sacralisent leurs structures » (Cosmao 1984, 4). C'est dire, bien sûr, que le rapport au Tiers et le rapport à l'autre se conditionnent mutuellement, dans un sens soit positif, soit négatif. Dans le premier cas, le mouvement paraît "descendant », du sacré aux rapports sociaux ou à un territoire dynamisé par la solidarité. Dans l'autre cas, selon un mouvement "ascendant ", l'autre est plutôt instrumentalisé par les intérêts de l'un au nom même du sacré dont il pervertit le rôle; il est en quelque sorte "sacrifié » à l'idole qui semble réclamer un tel geste. Le territoire luimême, c'est-à-dire les rapports tels qu'ils y prévalent, est alors sacralisé, rigidifié, empruntant au Tiers le trait de sa dégradation en simple garant du statu quo, plutôt que de faire évoluer son identité par l'ouverture. 


\section{Un traitement contextuel multidisciplinaire}

Terminons cette section par un regard sur le traitement du thème au plan des approches disciplinaires. C'est à dessein que ce numéro a pour tonalités dominantes celles de la science politique ${ }^{3}$, des sciences de la religion et, dans une moindre mesure, de la théologie. Mais l'anthropologie, la sociologie, la science économique, l'histoire et la philosophie y sont aussi convoquées dans des mesures variables. C'est que, d'une part, la transversalité des questions du territoire et du sacré comme de leurs rapports et, d'autre part, le caractère dramatique de leurs enjeux, enlèvent toute légitimité tant à une limitation des champs disciplinaires mis à contribution qu'à leur cloisonnement ${ }^{4}$. Les disciplines doivent être au service du savoir sur le réel et non l'inverse, ni projeter sur celui-ci leur propre découpage. S'impose alors un dialogue des disciplines avec toutes leurs connaissances accumulées et leurs procédés respectifs pour cerner la complexité du réel sans toutefois confondre les «langues». Chaque discipline n'a-t-elle pas besoin de laisser parler toutes les autres dans leur effort de dégagement des logiques à l'œuvre dans la réalité pour pouvoir mener son propre travail de façon fructueuse ? Une véritable approche contextuelle n'est pas à la mesure d'une seule discipline isolée. Ce dialogue, chaque auteur l'aura mené ici à sa façon, mais ce sera une tâche réservée surtout à chaque lecteur et lectrice en considérant l'ensemble de ces contributions.

\section{Les contributions à la thématique de ce numéro}

Il peut être utile, pour commencer, de dire un mot sur les « indications » de traitement qui avaient été proposées de prime d'abord aux auteurs, non pour les contraindre mais bien pour stimuler leur créativité. À propos des questions ou des problèmes d'ordre "politique» au sens le plus large, les auteurs étaient invités à un traitement sous l'angle de la structuration mutuelle entre le sacré et l'identité territoriale, en mettant en évidence la dimension même de processus, avec un accent soit sur la sacralisation du territoire, soit sur la territorialisation du sacré, ou les deux à la fois. Enfin,

3. Le rapport au territoire est au cœur de la science politique. Cette « jeune » discipline voit officiellement le jour à un moment et dans un monde où la logique étatique règne de façon suprême.

4. Ainsi, peut-on concevoir une science politique qui négligerait l'étude des logiques de sacralisation du pouvoir compris comme instance de coordination et de mise en société des humains qu'Aristote définissait comme des "animaux politiques " "par nature ", ou faits pour vivre dans la cité, en société ? (Aristote, Politique, I, 2, 9) 
le thème pouvait être abordé aussi bien selon une version proprement religieuse que selon une version séculière. Aucune étude purement théorique n'était sollicitée mais bien une réflexion contextuellement située. Le cas de Barros, à propos de la tradition biblique, faisait exception, mais nous savions déjà l'engagement de longue date de l'auteur auprès des paysans brésiliens sur les problèmes de la terre. Chaque auteur aura cependant su, en apportant sa touche propre, étayer son propos de considérations théoriques et de clarifications conceptuelles.

La séquence des cinq contributions obéit à la logique de la transformation du sacré dans le temps: d'abord le sacré religieux avec la tradition biblique (Barros); puis les réflexions de Prijatelj et McFalls sur le sacré national; ensuite Houtart, à propos du sacré économique dans la mondialisation néolibérale et de l'alternative à celle-ci; et, enfin, Doran, sur le sacré religieux traditionnel chez les Amérindiens Innus, expérience ancienne mais laissant paradoxalement entrevoir des éléments d'alternative aux impasses de la civilisation occidentale concernant le rapport au territoire et à l'environnement.

En ouverture, Barros évoque l'actualité des problèmes de la terre en Amérique latine pour les paysans et les indigènes mais aussi celle de la vision spirituelle des cultures autochtones pour relire l'évolution du rapport de la terre au sacré dans la Bible. Explorant d'abord le Premier Testament, il fait état du paradoxe du peuple hébreu rompant avec les cultes de la nature et de la terre, associés à ses dominateurs, pour quand même partager avec tout le Proche-Orient une culture religieuse où l'usufruit humain de la terre, terre sacrée parce qu'appartenant aux divinités symbolisées par des sanctuaires qui délimitent le territoire, reste indissociable du culte.

Mais des données nouvelles et singulières apparaissent dans l'itinéraire de ce peuple. La terre, ou territoire, n'est d'abord que "promise " par son Dieu sans terre, puis "donnée ", mais elle ne reste toujours que partiellement atteinte dans une quête constamment liée à l'observance de la Torah qui en règle l'utilisation en cohérence avec la volonté de celui à qui elle appartient en exclusivité. En un mot, le rapport à Dieu exige la justice sur sa terre considérée comme sa maison. C’est donc la Référence sacrée ellemême qui n'exerce sa souveraineté que pour assurer, par diverses mesures et limites, que son don soit partagé entre tous. C'est le sens des lois sabbatiques et jubilaires, par exemple, qui mettent un cran d'arrêt à l'ambition des latifundistes et qui obligent à des restitutions qui créent de nouveaux commencements. 
Au delà de toute distinction entre le profane et le sacré, la terre apparaît ainsi comme le premier "sacrement » de la promesse divine et comme «collaboratrice» de Dieu. Dans le Deuxième Testament, le Christ devient l'héritage fondamental de Dieu à son peuple, mais une terre de justice reste les prémices d'un Royaume qui nous invite à devenir les célébrants d'un «mariage» du ciel et de la terre. Le territoire, non plus donc que la Référence, n'est absolutisé, mais plutôt tiré vers l'avant, ouvert.

L'auteur appelle à une perspective "sacramentelle ou «enchantée ", dirions-nous, qui rompe avec cette séparation de l'humain et de la nature qui a mené à la marchandisation de la vie, et qui réintègre «la lutte pour la justice agraire à la justice socio-environnementale ». Le christianisme n'est-il pas interpellé ici à de nouveaux accents, plus bibliques, qui le dégagent des paradigmes qui, en nous soumettant aux idoles autoréférentielles du pouvoir et de l'argent, compromettent à la fois le sens de la réception de la terre comme don de Dieu et la possibilité qu'elle demeure un «territoire » hospitalier pour nos descendants?

Dans un contexte plus contemporain, mais qui n'en plonge pas moins ses racines dans une histoire millénaire évoquée d'abord à grands traits - histoire qui a vu le territoire slovène être amputé de plus des deux tiers et la plus grande partie de sa population se retrouver sous d'autres souverainetés et langues —, Erika Prijatelj nous propose une lecture de la crise identitaire de son pays. Après une exploration fouillée du sens du sacré, de la personne et de la nation, comme arrière-plan théorique et cadre d'analyse, la théologienne pose l'hypothèse que cette crise découlerait de la dénaturation des liens du nationalisme slovène avec le sacré. Partant du constat durkheimien que "de nombreuses communautés ont éprouvé le besoin de fondements sacrés, besoin exprimé par un culte, pour se situer dans l'existence » (Durkheim 1968, 5), Prijatelj analyse longuement l'impact de l'expérience totalitaire athée yougoslave sur l'identité slovène, une identité forgée par des liens profonds et étroits avec une religion que le communisme n'a cessé de vouloir éradiquer à tous les plans, tout comme le caractère inviolable de la dignité humaine, pour imposer de nouvelles formes de sacré, idéologique celui-là.

À son indépendance, en 1991, l'État slovène a revêtu des habits libéraux, mais malgré son entrée dans l'Union européenne, la nation slovène demeure encore mal définie. Prijatelj s'attelle à démontrer que le point névralgique de cette crise d'identité se situe dans la définition de l'ensemble des croyances qui rassemblent la communauté slovène et lui fournissent 
les ressources nécessaires à l'existence commune et à la survie des valeurs qui la font vivre. Si la moitié des Slovènes s'identifient à des valeurs religieuses chrétiennes, l'autre moitié les rejette. Par ailleurs, la lutte pour la libération nationale a perdu son statut de mythe rassembleur auprès d'une partie importante de la population. Restent la langue et la culture mais, selon Prijatelj, celles-ci ne suffisent pas. La nation slovène « en raison d'une plus grande fragilité reliée à son âge relativement jeune, a encore plus besoin que d'autres nations de protection vis-à-vis de l'individualisme, de la fragmentation, du relativisme, de l'apathie, de l'indifférence et du scepticisme postmodernes ». Détachés de leurs amarres identitaires historiques, les Slovènes se livrent "au polycentrisme des valeurs » et à la recherche «du plus petit dénominateur commun de convivialité sur leur territoire». Pour éviter les écueils identitaires de la mondialisation qui abat les frontières nationales et réorganise « en village global, des territoires, des nations et des continents », et qui, d'autre part, représente un défi à la spécificité nationale, - défi qui peut nourrir un nationalisme exacerbé mais également d'autres maux bien plus graves, dont la xénophobie, l'antisémitisme et diverses discriminations sociales - Prijatelj préconise le retour à un sacré rassembleur. Elle croit qu'un christianisme renouvelé pourrait y contribuer comme il a pu faire partie du socle de la civilisation européenne et aider grandement «les Slovènes à se développer sur leur territoire actuel ».

Cette analyse de l'expérience slovène interpelle vivement les nations qui, tel le Québec contemporain, ont connu un passé structuré par le christianisme, et qui tentent aujourd'hui de reprendre à neuf l'incontournable tâche historique de redéfinir dans une dialectique de toujours le tissu du sacré, du territoire et de leurs rapports.

Poursuivant dans la veine «nationaliste» du rapport du territoire au sacré, nous rencontrons ensuite un essai très original du politologue d'origine américaine Laurence McFalls sur l'expérience étatsunienne telle qu'analysée à partir du débat politique (ou de l'absence de débat) après le 11 septembre 2001. À travers la rebuffade essuyée dans sa correspondance personnelle avec un ami, intellectuel, l'auteur prend toute la mesure de la sacralisation de l' «états-unicité », qui va jusqu'à confondre le patriotisme avec la suspension de la liberté de réflexion, de jugement et de parole sur certains événements. Les attentats, qui ont atteint pour la première fois le territoire américain, ont été interprétés de façon hégémonique par la droite conservatrice, inspirée et soutenue par le fondamentalisme chrétien, comme une atteinte aux «prétentions transcendantes» de ce pays parmi les nations. 
Depuis toujours, le peuple américain se voit comme investi d'une mission divine, ayant la "destinée manifeste » d' «être une force de Bien dans le monde". Même la version la plus matérialiste du rêve américain, l'American way of life, fait aussi partie de cette référence sacrée, tout comme les visées impériales (Empire bienveillant, selon W. Kristol). Aussi, toute opposition à ce projet prend-elle immédiatement le sens du plus grand mal. Toute remise en question de cette représentation sacrée des États-Unis, serait-ce même à partir des faits, ou encore de l'acte de foi exigé à son égard, équivaut à un sacrilège qui ne rend plus son auteur « digne de la vie dans la cité».

Il paraît opportun, dans le contexte de cette analyse sur le pays le plus puissant et «avancé » du monde, d'écouter de nouveau Mircea Eliade nous parler de la Cité (moderne) et des accents de sa vision du monde qui l'apparentent encore à celle des sociétés dites traditionnelles: "S'il est vrai que "notre monde" est un Cosmos, toute attaque extérieure menace de le transformer en "Chaos" ». L'attaque de «notre monde» est l'œuvre de forces du Chaos et toute victoire contre un tel attaquant "réitère la victoire exemplaire du dieu contre le Dragon (contre le “Chaos”)» (1969, 47).

L'article suivant, du sociologue belge François Houtart, nous entraîne dans une nouvelle direction, à deux plans: celui d'un déplacement du sacré vers des valeurs profanes qu'il ne faut pas confondre avec la hiérophanie (Eliade 1969, 17), et celui d'une reterritorialisation du politique à l'économique.

Après avoir posé une problématique plus théorique du sacré et du territoire, à la fois en termes d'anthropologie fondamentale et d'économie politique en contexte international, l'auteur en montre le déploiement par une analyse au ras des transformations opérées par le capitalisme néolibéral mondialisé et par sa contrepartie altermondialiste. L'approche religiologique adoptée permet de cerner, là où on ne l'attendait pas, une nouvelle métamorphose du sacré et sa liaison étroite avec les enjeux territoriaux. La nouvelle référence sacrée et exclusive n'est autre que le marché autorégulateur, nouveau «dieu» au nom duquel de puissants intérêts défendent et légitiment l'appropriation privée et illimitée du territoire. Bousculant et rendant économiquement inopératoires les frontières politiques des territoires nationaux, les grands acteurs de la mondialisation néolibérale leur substituent un territoire propre, englobant et uniformisant la planète entière. Ils privatisent ou polluent les ressources communes même les plus essentielles (eau, air, patrimoine génétique, forêts, etc.) et colonisent l'ensemble des activités humaines et jusqu'au génome humain lui-même par leur 
marchandisation. À l'encontre du sacré «religieux» ou du nationalisme dont la référence ternaire unissait les humains dans un lien de solidarité communautaire, le Marché, référence à teneur binaire, généralise la logique de compétition et, conséquemment, d'exclusion. On y aura reconnu la corrélation entre un sacré idolâtrique et une sacrificialité destructrice des territoires humains.

L'altermondialisme, pour sa part, fait entrer en scène un nouvel acteur historique, la société civile, formée d'une «myriade d'organisations » autonomes qui résistent au néolibéralisme et qui cherchent à reterritorialiser le monde autrement, tant par des initiatives propres qu'en ramenant le marché et l'État, qu'il a satellisé, à leur raison d'être: le bien commun. Leur logique valorise le lien social et les cultures, le réseau et la diversité, et procède par convergence plutôt que par «une centralisation imposée de l'extérieur». L'auteur en décline le projet depuis l'utopie jusqu'aux mesures de court terme. Il met, enfin, en évidence la dimension spirituelle retrouvée de cette mouvance faisant profession d' «athéisme » face au sacré « réduit à la loi de la valeur », réfractaire à tout prosélytisme, mais ouverte aux références religieuses qui donnent souffle aux engagements en faveur des victimes et pour « un autre monde possible» et... nécessaire.

Dans un dernier article, l'anthropologue Anne Doran nous entraîne ailleurs, dans un autre monde, distant par sa culture, mais pourtant si proche géographiquement, et donc à la portée d'une rencontre possible, sinon nécessaire au vu du potentiel inespéré qu'il recèle pour nous faire passer le mur derrière lequel l'Occident a enfermé l'avenir commun.

Comment ne pas trahir ici une étude si minutieuse et si précieuse? L'auteur met d'abord en contraste la culture occidentale et la spiritualité des Innus quant à leurs rapports respectifs au monde. Elle poursuit ensuite ce dépaysement en explorant à fond comment la compréhension du monde et l'identité innue se fondent sur le lien à un territoire. À l'encontre d'un Occident se comprenant dans l'opposition à la nature vue comme simple ressource à s'approprier et exploiter, le monde, qui « est territoire » et dont l'humain fait partie intégrante, est perçu dans la culture traditionnelle innue comme don et lieu de la présence de l'Être, et donc comme reçu et comme sacré. Cette « reconnaissance de l'élément spirituel qui habite chaque être et en fonde l'irréductible individualité » fait entrer l'humain "en relation de don et de contre-don avec les éléments du cosmos pour soutenir sa vie et permettre en même temps au grand tout [...] de poursuivre la sienne ». N'y a-t-il pas là toute la différence du monde entre le village, ou pire, la 
«réserve », et la forêt ou le territoire de chasse comme «lieu» de la communauté innue?

Cette cosmovision implique une logique d'inclusion dans un territoire organisé «en fonction d'un partenariat entre humains et non-humains ». Le don en est le sens et la cheville ouvrière, le rouage essentiel. Le lien social qu'il entretient s'inscrit dans une réciprocité généralisée entre tous les êtres. Nous avons ici une ternarité positive exemplaire: un mouvement de don issu de la Référence (ici, sacrée) entraîne une solidarité constituante d'un territoire comme milieu commun insubstituable de circulation de la vie sous la responsabilité de tous. Comment ne pas entendre ici cette parole de l'évangile: "Vous avez reçu gratuitement, donnez gratuitement " (Mt 10, 8). Ou encore son écho chez Bernanos: "Mais la vie m'enseigne [...] que nous ne recevons rien que nous n'ayons d'abord donné. Entre nous il n'est qu'échange. Dieu seul donne, lui seul » (1949, 787).

Sans qu'il s'agisse de nous couler entièrement dans une culture ou une spiritualité si étrangères à notre occidentalité, des ponts pourraient cependant être jetés et une convergence être tentée. L'auteur s'attarde ainsi aux possibilités de reconsidération des rapports à la nature et au territoire dans un monde où la gestion de l'environnement soulève un problème des plus pressants. Elle en appelle aussi à un regard théologique critique au vu du double drame vécu à cet égard par les Innus à l'époque de leur «colo-évangélisation ». D’une part, le christianisme des missionnaires a déconsidéré ou démonisé la capacité de dévoilement par le monde de la réalité spirituelle, oublieux de la vision patristique étendant aux dimensions de l'univers ( " plein de mystères ", selon Origène) les limites de la corporéité du Ressuscité. D'autre part, ce christianisme, obsédé par «l'échec de la création » sous le péché et par une rédemption sans elle, avait trahi la ternarité foncière de la foi chrétienne en délestant le rapport à Dieu du rapport au monde. Ou plutôt, refusant le "prophétisme » du rapport innu au monde, cette théologie a cherché à lui substituer le rapport imposé par le colonisateur, certes pas politiquement neutre!

Cédons donc maintenant la place à nos auteurs mêmes, qui ont pris le risque d'une parole sur une question difficile mais importante. En espérant que cette lecture entame d'abord avec eux et avec elles, puis avec d'autres interlocuteurs et interlocutrices, un dialogue essentiel dans la quête commune de références aptes à favoriser le vivre ensemble sur nos «territoires » et, à ce titre seulement, dignes de quelque révérence «sacrée ». 


\section{Références}

Aglietta, M. et A. Orlean (1982), La violence de la monnaie, Paris, PUF (Économie en liberté).

ANDERson, B. (1993), Imagined Communities. Reflections on the Origin and Spread of Nationalism, Londres, Verso.

Aristote, Politique, Livre I.

Bernanos, G. (1949), Les enfants humiliés, dans Essais et écrits de combat, t. 1, Paris, Gallimard (Bibliothèque de la Pléiade).

Cosmao, V. (1981), Changer le monde, une tâche pour l'Église, Paris, Cerf (Traditions chrétiennes).

(1984), «Problématique de la théologie de la libération », Foi et développement, 117-118, p. 1-6.

Despland, M. (1979), La religion en Occident. Évolution des idées et du vécu, Montréal, Fides (Héritage et projet, 23).

DORY, D. (1989), «Territoire », dans A. JACOB, dir., Encyclopédie philosophique universelle, vol. 2 Notions philosophiques - Dictionnaire, t. 2, $M-Z$, Paris, PUF, p. 2574-2575.

Dufour, D.-R. (1990), Les mystères de la trinité, Paris, Gallimard. (2003), L'art de réduire les têtes. Sur la nouvelle servitude de l'homme libéré à l'ère du capitalisme total, Paris, Denoël.

DuPuY, J.-P. (1988), "L'homme machine et les adorateurs du signifiant », Le Débat, 49, p. 164-184.

DuRKheIM, E. $\left(1968^{5}\right)$, Les formes élémentaires de la vie religieuse. Le système totémique en Australie, Paris, PUF (Bibliothèque de philosophie contemporaine).

Éliade, M. (1969), Le sacré et le profane, Paris, Gallimard (Idées).

FukuYama, Francis (1992), The End of History and the Last Man, New York, Avon Books.

GIRARD, R. (1978), Des choses cachées depuis la fondation du monde, Paris, Grasset.

Legendre, P. (1998), La 901e conclusion. Étude sur le théâtre de la Raison, Paris, Fayard.

LOCKe, J. (1990), Traité du gouvernement civil, trad. D. Mazel (1795), à partir du texte de la $5^{\mathrm{e}}$ édition de Londres publiée en 1728, Paris, Garnier-Flammarion (Texte intégral). 
Mueller, J. (1989), Retreat from Doomsday. The Obsolescence of Major War, New York, Basic Books.

Ost, F. (1999), Du Sinaï au Champ-de-Mars. L'autre et le même au fondement du droit, Bruxelles, Lessius (Donner raison).

Oтто, R. (1949), Le sacré, Paris, Payot.

PAtez, F. (1998), "Quelques remarques sur l'imaginaire national », Les Cahiers du Cériem, 3, p. 3-16.

RuFIN, J.-C. (2004), Globalia, Paris, Gallimard.

Ruthven, M. (2004), Fundamentalism. The Search for Meaning, Oxford, Oxford University Press.

Texier, R. (1989), "Sacré», dans A. JACOB, dir., Encyclopédie philosophique universelle, vol. 2 Notions philosophiques - Dictionnaire, t. 2, M-Z, Paris, PUF, p. 2291-2293. 